\title{
Solutions with Dead Cores for a Parabolic P-Laplacian Equation
}

\author{
Zhengce Zhang, Yanyan Li \\ College of Science, Xi' an Jiaotong University, Xi' an, China \\ E-mail:zhangzc@mail.xjtu.edu.cn, liyan86911@126.com \\ Received June 10, 2011; revised July 15, 2011; accepted July 25, 2011
}

\begin{abstract}
We study the solutions with dead cores and the decay estimates for a parabolic p-Laplacian equation with absorption by sub- and supersolution method. Special attention is given to the case where the solution of the steady-state problem vanishes in an interior region.
\end{abstract}

Keywords: Parabolic P-Laplacian Equation, Steady State, Dead Core, Decay Estimates

\section{Introduction}

In this paper, we study the following initial-boundary value problem for $u(x, t)$ :

$$
\left\{\begin{array}{l}
u_{t}-\operatorname{div}\left(|\nabla u|^{p-2} \nabla u\right)=-\lambda u^{q} \quad \text { in } Q=\Omega \times \mathbb{R}^{+}, \\
u(x, t)=\chi(x) \geq 0 \quad \text { on } \Gamma=\partial \Omega \times \mathbb{R}^{+}, \\
u(x, 0)=u_{0}(x), \quad 0 \leq u_{0}(x) \leq 1 \quad \text { in } \Omega,
\end{array}\right.
$$

with $\lambda \in \mathbb{R}^{+}, p>1$ and $0<q<p-1$. Here $\chi(x)$ is continuous on $\Gamma$ and $u_{0}(x)$ can be extended to a function on $\bar{\Omega}$, satisfying the compatibility condition

$$
u_{0}(x)=\chi(x), \quad x \in \partial \Omega .
$$

The domain $\Omega \subseteq \mathbb{R}^{N}(N>1)$ is smooth and bounded.

Our purpose is to describe how the solution $u(x, t)$ of (1.1) tends to its steady state and the existence of dead cores. A dead core $\Omega_{0}:=\{x \in \Omega: u(x)=0\}$, i.e. a region where the solution vanishes identically may appear. Such a region is a waste from the engineering point of view. We concern its existence. Our method is the weak solution which is similar to that in [4] where the porous medium equation was considered. For the case of $1<p<2$ and $q>1$ of the problem (1.1), Chen, Qi and Wang [3] proved the existence of the singular solution. In [2], they also studied the long time behavior of solutions to the Cauchy problem of

$$
u_{t}=\operatorname{div}\left(|\nabla u|^{p-2} \nabla u\right)-u^{q} \quad \text { in } \mathbb{R}^{N} \times(0, \infty)
$$

with nonnegative initial value $u(x, 0)=\varphi(x)$ in $\mathbb{R}^{N}$, where $2 N /(N+1)<p<2$ and $q>1$. For initial data of various decay rates, especially the critical decay $\varphi=O\left(|x|^{-\mu}\right)$ with $\mu=p /(q+1-p)$, they showed that the solution converges as $t \rightarrow \infty$ to a self-similar solution.

We have known the following behavior of the absorption near $u=0$ :

$$
\begin{gathered}
q<1 \quad \text { strong absorption, } \\
q \geq 1 \quad \text { weak absorption. }
\end{gathered}
$$

When $\chi(x) \equiv 0$ in (1.1), the steady state vanishes and $u(x, t)$ tends to zero as $t \rightarrow \infty$. Strong absorption yields extinction in finite time, that is, there is a time $T$ such that $u(x, t) \equiv 0$ for all $x$ and $t \geq T$. For small $u$, the absorption is still relatively large in the case of strong absorption and will tend to drive the solution more quickly to zero than in the case of weak absorption. If $\chi(x)$ is not identically zero in (1.1), the corresponding steady state $\phi(x)$ does not vanish identically, but it is still true that $u(x, t)$ tends to $\phi(x)$ as $t \rightarrow \infty$.

The steady-state problem

$$
\begin{aligned}
& -\operatorname{div}\left(|\nabla \phi|^{p-2} \nabla \phi\right)=-\lambda \phi^{q} \text { in } \Omega, \\
& \phi(x)=\chi(x) \text { on } \partial \Omega
\end{aligned}
$$

is a special case of the problem

$$
\begin{aligned}
& \sum_{i=1}^{N}\left(a(x)|\nabla u|^{p-2} u_{x_{i}}\right)_{x_{i}}=b(x) f(u) \text { in } D, \\
& u=1 \text { on } \partial D
\end{aligned}
$$

in [1], where $D \in \mathbb{R}^{N}$ be an arbitrary domain, $a, b: D \rightarrow \mathbb{R}^{+}$be two continuous functions, $p \geq 1$ and $f(s)$ is a nondecreasing function with $f(0)=0$. 
From [1], we know that Problem (1.2) has a unique solution, a dead core exists if $\lambda$ is large enough and

$$
\int_{0}^{1} F^{-1 / p}(s) \mathrm{d} s<\infty,
$$

where $F(s)=\int_{0}^{s} f(\xi) \mathrm{d} \xi$.

Proofs of existence and uniqueness for Problem (1.1) are based on a suitable notion of weak solution, which we include here for the sake of completeness.

Definition 1.1 Let $Q_{T}=\Omega \times(0, T)$ and let $n$ denote the outward unit normal to $\Omega$. A function $u(x, t) \in C\left(W^{1, p}(\Omega) \cap L^{\infty}\left(Q_{T}\right) ;[0, T]\right)$ is called a weak solution of Problem (1.1) if it satisfies

$$
\begin{aligned}
& \int_{\Omega} u(x, T) \sigma(x, T) \mathrm{d} x \\
& +\int_{Q_{T}}\left[|\nabla u|^{p-2} \nabla u \cdot \nabla \sigma-u \sigma_{t}\right] \mathrm{d} x \mathrm{~d} t \\
& -\int_{0}^{T} \int_{\partial \Omega}|\nabla \chi|^{p-2} \frac{\partial \chi}{\partial n} \sigma \mathrm{d} s \\
& =\int_{\Omega} u_{0} \sigma(x, 0) \mathrm{d} x-\lambda \int_{Q_{T}} u^{q} \sigma \mathrm{d} x \mathrm{~d} t
\end{aligned}
$$

for all $\sigma \in C^{1}\left(\bar{Q}_{T}\right)$ with

$$
\sigma(x, t)-\chi(x, t) \in C\left(W_{0}^{1, p}(\Omega) \cap L^{\infty}\left(Q_{T}\right) ;[0, T]\right) .
$$

Equation (1.5) is obtained easily by multiplying (1.1) by $\sigma$, integrating over $Q_{T}$, and using the divergence theorem.

A weak supsolution of (1.1) is defined by replacing the equal sign in (1.5) by $\geq$ and restricting $\sigma$ to be nonnegative. Similarly, we can define a weak subsolution. For our purposes, it suffices to consider the usual super- and subsolutions defined as follows.

We say that $\bar{u} \geq 0$ is a supersolution of (1.1) if

$$
\begin{aligned}
& \bar{u}_{t}-\operatorname{div}\left(|\nabla \bar{u}|^{p-2} \nabla \bar{u}\right)+\lambda \bar{u}^{q} \geq 0, \\
& \bar{u}(x, 0) \geq u_{0}(x),\left.\quad \bar{u}\right|_{\partial \Omega} \geq \chi(x) .
\end{aligned}
$$

Similarly, $\underline{u} \geq 0$ is a subsolution if all the inequalities in (1.6) are reversed. If $\underline{u} \leq \bar{u}$, then the unique weak solution $u$ of (1.1) satisfies $\underline{u} \leq u \leq \bar{u}$, almost everywhere in $Q_{T}$.

It follows from the maximum principle that the solutions of (1.1) and of (1.2) satisfy $u(x, t) \leq 1$ in $Q$, $\phi(x) \leq 1$ in $\Omega$.

To show monotonicity of $u(x, t)$ in time, we need to impose a natural condition on the initial value $u_{0}(x)$ :

$$
\operatorname{div}\left(\left|\nabla u_{0}(x)\right|^{p-2} \nabla u_{0}(x)\right)-\lambda u_{0}^{q} \leq a<0, \quad x \in \Omega .
$$

This condition holds automatically if $u_{0}(x)$ is a positive constant. If $u_{0}(x)$ satisfies (1.7), it is an upper solution to (1.1) so that $u(x, t) \leq u_{0}(x)$ for any $t$. Now let $v(x, t)=u(x, t+\tau)$; then $v(x, t)$ satisfies

$$
\begin{aligned}
& v_{t}-\operatorname{div}\left(|\nabla v|^{p-2} \nabla v\right)=-\lambda v^{q}, \\
& v(x, 0)=u(x, t), \quad v(\partial \Omega, t)=\chi(x) .
\end{aligned}
$$

Since $u(x, \tau) \leq u_{0}(x), \quad v$ is subsolution of (1.1), and hence $u(x, t+\tau) \leq u(x, t)$. Hence, $u(\cdot, t)$ is monotonically decreasing. Standard theorems can be used to show that $u(x, t)$ tends to the steady-state $\phi(x)$ as $t \rightarrow \infty$.

\section{Monotonicity and Other Comparison Theorems}

Consider problem (1.1) when only one part of the data is changed. We then have the following monotonicity properties:

1) Let $u_{1}$ and $u_{2}$ be the solutions corresponding to $\lambda_{1}, \lambda_{2}$, respectively, with $\lambda_{1} \leq \lambda_{2}$; then $u_{2} \leq u_{1}$ in $Q$.

2) If the initial or the boundary value is decreased so is the solution.

3) Let $u_{0}(x) \equiv 1, \quad \chi(x) \equiv 1$ and consider two domains $\Omega_{1} \subset \Omega_{2}$. Then $u_{2} \leq u_{1}$ on $Q_{1}$.

These all are easy to prove using super- and subsolution techniques. Here we omit the proofs.

Next we look at the "lumped-parameter" problem and the steady-state problem with a view to using them as comparison problems for (1.1). The lumped parameter problem has no diffusion term. It can be obtained as a special case of (1.1) with initial value $u_{0}(x) \equiv 1$ and boundary condition of vanishing normal derivative. We can then seek a solution $z(t)$ independent of $x$ :

$$
z_{t}=-\lambda z^{q}, \quad t>0 ; \quad z(0)=1 .
$$

The solution is given explicitly by

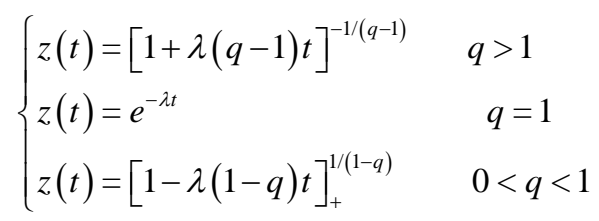

Therefore, extinction occurs in finite time if and only if $0<q<1$. Then $\lambda t=1 /(1-q)$ and $z(t)>0$ for $\lambda t<1 /(1-q)$.

Comparison with (2.1) leads immediately to two results for (1.1).

Theorem 2.1 (a) If $\chi(x)=0$, then $z(t)$ is a supersolution of (1.1), so that $u(x, t) \leq z(t)$ and, if the absorption is strong, there is extinction in finite time for $u(x, t)$.

(b) If $\min _{\bar{\Omega}} u_{0}(x)=\theta>0$, then the solution $z(t, \theta)$ of (2.1) with initial value $\theta$ is a subsolution of (1.1) so that $u(x, t) \geq z(t, \theta)$. If the absorption is weak, then $z(t, \theta)>0$ for all $t$, and hence $u(x, t)>0$ in $Q$.

The following theorem shows that (1.4) is necessary and sufficient for the existence of a dead core for 
sufficiently large $\lambda$.

Theorem 2.2 For $0<q<p-1, p>1$, (1.4) is satisfied. Then a dead core exists for sufficiently large $\lambda$.

Proof. We shall construct, for $\lambda$ sufficiently large, an upper solution $v(r)$ to (1.2) for a ball $B_{R}$, with $v \equiv 0,0<r<R / 2$. We begin by observing that on the positive real line, the function $w(x)$ by

$$
\begin{aligned}
& w(x)=\left(\left(\frac{p}{p-1-q}\right)\right)^{p /(p-1-q)} \\
& \left(\frac{p \mu}{(1+q)(p-1)}\right)^{p /(p-1-q)} x^{\frac{p}{p-1-q}}
\end{aligned}
$$

satisfies $(p-1)\left(w^{\prime}\right)^{p-2} w^{\prime \prime}=\mu w^{q}, \quad x>0$;

$w(0)=w^{\prime}(0)=0$. Now we choose $\mu$ so that

$w\left(\frac{R}{2}\right)=1$ and consider the function

$$
v=w\left(r-\frac{R}{2}\right)
$$

in the ball $r \leq R$ in $\mathbb{R}^{N}$ (having extend $v$ to be zero for $r<R / 2$ ). We then obtain

$$
\begin{aligned}
& \operatorname{div}\left(|\nabla v|^{p-2} \nabla v\right)=(p-1)\left(v^{\prime}\right)^{p-2} v^{\prime \prime}+\frac{N-1}{r}\left(v^{\prime}\right)^{p-1} \\
& =\mu v^{q}+\frac{(N-1)}{r}\left(v^{\prime}\right)^{p-1} \text { in }(R / 2, R) \\
& =\mu v^{q}+\frac{(N-1)}{r}\left(\left(\frac{p \mu}{(1+q)(p-1)}\right)^{1 / p} v^{(1+q) / p}\right)^{p-1} \\
& \leq \mu v^{q}+C v \frac{(1+q)(p-1)}{p} \\
& \leq(\mu+C) v^{q}:=v v^{q},
\end{aligned}
$$

where we use $0 \leq v \leq 1$ and $\frac{(1+q)(p-1)}{p} \geq q$. Since $v(R / 2)=0, v^{\prime}(R / 2)=0$, the last inequality can be extended to $(0, R)$. Furthermore, $v(R)=1$ so that $v(r)$ is an upper solution of the elliptic problem (1.2) for $\lambda \geq v$. Since $v$ vanishes for $r<R / 2$, so does $\phi$. Now consider (1.2) on an arbitrary domain $\Omega$ with $\phi(\partial \Omega) \leq 1$. Then $\Omega$ contains a ball $B_{R}$ on whose boundary $\phi \leq 1$. Therefore, $\phi \leq v$ and $\Omega$ contains a dead core for $\lambda \geq v$.

For any $x_{0} \in \Omega$, we can take $R=r_{0}=$ the distance from $x_{0}$ to the boundary. Theorem 2.2 shows that for $\lambda$ large enough, $x_{0}$ belongs to the dead core. This suggests making the following definition.

Definition 2.1 Let $x_{0} \in \Omega$. Define

$$
\lambda_{0}=\inf _{\lambda}\left\{\phi\left(x_{0}, \lambda\right)=0\right\} ; \lambda^{*}=\inf _{x_{0}} \lambda_{0} .
$$

We claim that

$$
\lambda_{0} \leq \frac{P_{N, p, q}}{r_{0}^{p}}, \quad \lambda^{*} \leq \frac{P_{N, p, q}}{\rho^{2}},
$$

where $P_{N, p, q}=(p-1)\left(\frac{p}{p-1-q}\right)^{p-1}\left(\frac{p}{p-1-q}+N-2\right)$, $r_{0}=\operatorname{dist}\left(x_{0}, \partial \Omega\right) ; \rho=$ inradius of $\Omega$. The proof consists in noting that the function $w=\left(\frac{\left|x-x_{0}\right|}{r_{0}}\right)^{p /(p-1-q)}$ satisfies $-\operatorname{div}\left(|\nabla w|^{p-2} \nabla w\right)+\frac{P_{N, p, q}}{r_{0}^{p}} w^{q}=0$ in $\Omega$ and $w(\partial \Omega) \geq 1$. Thus, $w$ is a supersolution of (1.2) for any $\lambda \geq \frac{P_{N, p, q}}{r_{0}^{p}}$. Since $w$ vanishes at $x_{0}$, so does $\phi$. This proves the first part of (2.4) and the second part follows at once.

\section{The Corresponding Evolution Problem}

Since (1.4) is satisfied with $\lambda$ large enough, the steady state has a dead core. Does the corresponding evolu- tion problem have a dead core and, if so, how does it behave for large $t$ ? The answer is given by the fol- lowing theorem.

Theorem 3.1 For fixed $x_{0} \in \Omega$, choose $\lambda>\lambda_{0}$, where $\lambda_{0}$ is defined in (2.3). Then

(a) if $0<q<p-1 \leq 1, u\left(x_{0}, t\right)=0$ for

$$
t \geq t_{0}:=\frac{1}{(1-q)\left(\lambda-\lambda_{0}\right)}
$$

(b) if $1 \leq q<p-1$ and $\min _{\bar{\Omega}} u_{0}(x)>0$, then $u\left(x_{0}, t\right)>0$ for all $t$.

Proof. Part (b) is equivalent to Theorem 2.1(b). To prove part (a), it suffices to exhibit a supersolution $w(x, t)$ such that $w\left(x_{0}, t\right)=0$ for $t \geq t_{0}$. We try a function

$$
w=z+\phi,
$$

where $z(t, \gamma)$ is the solution of the lumped--parameter problem with $\gamma$ to be choosen satisfies the differential inequality for a supersolution and $\phi$ is the solution of the steady-state problem with $\lambda=\lambda_{0}$. Note that $w$ vanishes at $x_{0}$ for $\gamma t \geq 1 /(1-q)$. Since $w \geq z$ and $w \geq \phi$, it is clear that $w(x, 0) \geq u_{0}(x)$ and $w(\partial \Omega, t) \geq \chi(x)$. From the definition of $w$, we have

$$
\begin{aligned}
& w_{t}-\operatorname{div}\left(|\nabla w|^{p-2} \nabla w\right)+\lambda w^{q} \\
& =z_{t}-\operatorname{div}\left(|\nabla \phi|^{p-2} \nabla \phi\right)+\lambda(z+\phi)^{q} \\
& \geq-\gamma z^{q}-\lambda_{0} \phi^{q}+\lambda(z+\phi)^{q} \geq\left(\lambda-\gamma-\lambda_{0}\right) w^{q} .
\end{aligned}
$$


By choosing $\gamma=\lambda-\lambda_{0}$, we obtain the desired result.

\section{Decay Estimates}

We consider (1.1) with $u_{0}(x) \geq \phi(x)$, where $\phi(x)$ is the solution of the corresponding steady-state problem (1.2). It then follows, since $\phi$ is a lower solution, that $u(x, t) \geq \phi(x)$. If we assume in addition that $u_{0}(x) \in L^{\infty}(\Omega)$, then $u$ is bounded a.e. in $Q_{\infty}$.

Our estimates hold for almost all $x \in \Omega$. If the data are smooth, the solution is continuous and the estimates hold pointwise. We seek decay estimates for $\delta(x, t)=u(x, t)-\phi(x)$. Our principal results are contained in the following theorem.

Theorem 4.1 (a) if $1 \leq q<p-1$, then

$$
0 \leq \delta(x, t) \leq z(t),
$$

where $z(t)$ is the solution of (2.1).

(b) if $0<q<p-1 \leq 1$, then

$$
0 \leq \delta(x, t) \leq \zeta(t),
$$

where $\zeta(t)$ is the solution of

$$
\zeta_{t}=-\gamma \zeta, \zeta(0)=1, \gamma=\lambda q 2^{q-1} .
$$

Proof. (a) We choose $w(x, t)=z(t)+\phi(x)$, where $z(t)$ satisfies (2.1) and $\phi(x)$ satisfies (1.2). Then, we have

$$
\begin{aligned}
& w_{t}-\operatorname{div}\left(|\nabla w|^{p-2} \nabla w\right)+\lambda w^{q} \\
& =z_{t}-\operatorname{div}\left(|\nabla \phi|^{p-2} \nabla \phi\right)+\lambda w^{q} \\
& =z_{t}-\lambda \phi^{q}+\lambda w^{q} \\
& \geq-\lambda z^{q}-\lambda u^{q}+\lambda\left(z^{q}+\phi^{q}\right)=0,
\end{aligned}
$$

where we use $q \geq 1$. Because we also have $w \geq z$ and $w \geq \phi$, we see that $w$ is a supersolution of (1.1). Then

$$
\delta=u-\phi \leq w-\phi=z .
$$

(b) We choose $v(x, t)=\zeta(t)+\phi(x)$, where $\zeta(t)$ satisfies (4.1) and $\phi$ satisfies (1.2). Then, we have

$$
\begin{aligned}
& v_{t}-\operatorname{div}\left(|\nabla v|^{p-2} \nabla v\right)+\lambda v^{q} \\
& =\zeta_{t}-\operatorname{div}\left(|\nabla \phi|^{p-2} \nabla \phi\right)+\lambda v^{q} \\
& =\lambda\left[(\zeta+\phi)^{q}-\phi^{q}\right]+\zeta_{t} \\
& \geq \lambda q(\zeta+\phi)^{q-1} \zeta-\gamma \zeta \\
& =\left(\lambda q(\zeta+\phi)^{q-1}-\gamma\right) \zeta \geq 0 .
\end{aligned}
$$

Because we also have $v \geq \zeta$ and $v \geq \phi$, we see that $v$ is a supersolution of (1.1). Then

$$
\delta=u-\phi \leq v-\phi=\zeta .
$$

Theorem 4.1 is proved.

\section{Acknowledgments}

The authors are grateful to the Fundamental Research Funds for the Central Universities of China.

\section{References}

[1] C. Bandle and S. Vernier-Piro, "Estimates for Solutions of Quasilinear Problems with Dead Cores,” Zeitschrift für Angewandte Mathematik und Physik, Vol. 54, No. 5, 2003, pp. 815-821.

[2] X. F. Chen, Y. W. Qi and M. X. Wang, "Long Time Behavior of Solutions to P-Laplacian Equation with Absorption," SIAM Journal on Mathematical Analysis, Vol. 35, No. 1, 2003, pp. 123-134. doi:10.1137/S0036141002407727

[3] X. F. Chen, Y. W. Qi and M. X. Wang, "Singular Solutions of Parabolic P-Lapacian with Absorption," Transactions of the American Mathemarical Society, Vol. 359 2007, pp. 5653-5668.

[4] C. Bandle, T.Nanbu, I. Stakgold, "Porous Medium Equation with Absorption," SIAM Journal on Mathematical Analysis, Vol. 29, 1998, pp. 1268-1278. doi:10.1137/S0036141096311423 